\title{
A Comparative Study of Particle Size Distribution of Graphene Nanosheets Synthesized by an Ultrasound-Assisted Method
}

\author{
Juan Amaro-Gahete ${ }^{1,+}{ }^{\oplus}$, Almudena Benítez ${ }^{2,+}\left(\mathbb{D}\right.$, Rocío Otero $^{2}$, Dolores Esquivel ${ }^{1}(\mathbb{D}$, \\ César Jiménez-Sanchidrián ${ }^{1}$, Julián Morales ${ }^{2}$, Álvaro Caballero ${ }^{2, *} \mathbb{B}$ and \\ Francisco J. Romero-Salguero ${ }^{1, *}$ (i) \\ 1 Departamento de Química Orgánica, Instituto Universitario de Investigación en Química Fina y \\ Nanoquímica, Facultad de Ciencias, Universidad de Córdoba, 14071 Córdoba, Spain; \\ q22amgaj@uco.es (J.A.-G.); q12esmem@uco.es (D.E.); qo1jisac@uco.es (C.J.-S.) \\ 2 Departamento de Química Inorgánica e Ingeniería Química, Instituto Universitario de Investigación en \\ Química Fina y Nanoquímica, Facultad de Ciencias, Universidad de Córdoba, 14071 Córdoba, Spain; \\ q62betoa@uco.es (A.B.); b42otizr@uco.es (R.O.); iq1mopaj@uco.es (J.M.) \\ * Correspondence: alvaro.caballero@uco.es (A.C.); qo2rosaf@uco.es (F.J.R.-S.); Tel.: +34-957-218620 (A.C.) \\ + These authors contributed equally to this work.
}

Received: 24 December 2018; Accepted: 23 January 2019; Published: 26 January 2019

\begin{abstract}
Graphene-based materials are highly interesting in virtue of their excellent chemical, physical and mechanical properties that make them extremely useful as privileged materials in different industrial applications. Sonochemical methods allow the production of low-defect graphene materials, which are preferred for certain uses. Graphene nanosheets (GNS) have been prepared by exfoliation of a commercial micrographite (MG) using an ultrasound probe. Both materials were characterized by common techniques such as X-ray diffraction (XRD), Transmission Electronic Microscopy (TEM), Raman spectroscopy and X-ray photoelectron spectroscopy (XPS). All of them revealed the formation of exfoliated graphene nanosheets with similar surface characteristics to the pristine graphite but with a decreased crystallite size and number of layers. An exhaustive study of the particle size distribution was carried out by different analytical techniques such as dynamic light scattering (DLS), nanoparticle tracking analysis (NTA) and asymmetric flow field flow fractionation (AF4). The results provided by these techniques have been compared. NTA and AF4 gave higher resolution than DLS. AF4 has shown to be a precise analytical technique for the separation of GNS of different sizes.
\end{abstract}

Keywords: graphene nanosheets; exfoliation; particle size distribution; nanoparticle tracking analysis; asymmetric flow field flow fractionation

\section{Introduction}

Particle size analysis is a key element because many properties of nanomaterials are size dependent [1]. This parameter is essential since the synthesis at a small scale must be monitored for subsequent bulk production and for the control of nanotechnological products in the market. The control of size during the synthesis of nanomaterials is decisive in various industrial sectors such as nanomedicine, nanofood, nanoenergy or nanocosmetics [2-6].

It is well known that graphene has excellent properties that provide a multitude of technological applications in different fields. It is formed by a pattern of hexagonal rings of carbon atoms constituting a huge flat molecule [7]. Some of its most important properties are its high thermal and electrical 
conductivity, its high elasticity, its hardness and strength and its flexibility, in that it is more flexible than carbon fibre and equally lightweight [8].

Many methods for obtaining graphene have been reported. Graphene layers can be obtained by liquid-phase exfoliation of commercial graphite in different organic solvents using the ultrasonic technique. This method requires special experimental conditions at which very high pressures and temperatures are reached in very short periods of time [9]. The colloidal graphene generated has the same surface energy as the solvent used. In this way, the free energy is negative, and the structure is broken, giving rise to fragments of graphite interspersed with solvent molecules [10]. For the successful exfoliation of graphite, different organic solvents have been used, such as N-methyl pyrrolidone (NMP), dimethylformamide (DMF), pyridine and $o$-dichlorobenzene (ODBC) [11,12]. By means of the ultrasound method, a maximum of $15 \%$ of individual graphene sheets is obtained. The rest is a mixture of multilayer graphene or few-layer graphene. A very important parameter for obtaining few-layer graphene is to carry out a selective centrifugation [13]. By carrying out this operation it is possible to separate the non-exfoliated graphitic particles from the exfoliated graphene sheets. This method of synthesis has different advantages with respect to conventional graphene production methods: strong oxidizing agents are not required, the synthesis time is reduced and unfunctionalized and non-oxidized graphenes are obtained in one step [14]. On the contrary, this process can have negative effects, since prolonged sonication times can facilitate the existence of defects in the surface of the graphene sheets and the reduction of the layer size [15]. For instance, these problems have been observed when DMF is used as an exfoliating liquid phase [16]. For this reason, it is very important to control the sonication times during the exfoliation process of the graphite and, above all, to choose the solvent that generates the least problems [17].

$o$-Dichlorobenzene is an organic solvent that has excellent properties to be used as liquid phase for the process of graphite exfoliation. It is commonly applied as a reaction medium in fullerene chemistry and it is well known that it forms very stable dispersions due to its very efficient interactions with graphene via $\pi-\pi$ stacking [18]. On the other hand, it has a high boiling point and a surface tension suitable for the correct exfoliation [12].

Graphene-based materials have been characterized by a variety of different techniques, such as X-ray diffraction, transmission electron microscopy and Raman spectroscopy, among others $[19,20]$. Although these techniques can provide some information about the size of these materials, dynamic light scattering (DLS) is particularly useful to determine particle size. Thus, the use of DLS to measure the size of graphene nanosheets and exfoliated graphene oxide has been reported as a simple and fast method of characterization [21]. This technique is based on the Brownian motion of the particles in suspension causing the scattering of light at different time-dependent fluctuations intensities. These fluctuations are directly related to the diffusion coefficient of the particles in the solvent, which provides in turn the particle size using the Stokes-Einstein relationship. However, it is well known that this technique is more reliable for spherical particles than for non-spherical particles [22]. Moreover, if a sample is polydisperse, as usually happens for graphene nanomaterials prepared by most methods, DLS will exhibit some limitations to measure particle size (vide infra) [23]. Furthermore, two techniques have emerged in the last years for the characterization of nanoparticles, i.e., nanoparticle tracking analysis (NTA) and asymmetric flow field-flow fractionation (AF4). To the best of our knowledge, none of them has been used for the characterization of graphene-based materials.

NTA is a relatively novel technique for the study of particle size distribution. Like DLS, NTA uses light scattering and the Brownian motion of liquid suspensions of particles to obtain the size distribution in a sample. This technique allows real-time visualization and recording of nanoparticles during the measurement by combining a charge-coupled device with laser light scattering microscopy [24]. In this way, the NTA software tracks individual particles and, using a formula derived from the Stokes-Einstein equation, provides particle size values by calculating the particle hydrodynamic diameter. In addition, it gives other parameters such as particle concentration, the range of which must be $10^{6}$ to $10^{9}$ particles $/ \mathrm{mL}$, aggregation and fluorescence [25]. The concentration 
of particles present in the sample does not depend on the scattered light, so this technique allows an accurate and reproducible determination of the concentration of non-spherical particles as in the case of carbon nanotubes or colloids [26]. Based on these characteristics, NTA is frequently used to accurately obtain the size of protein aggregates and drug delivery nanoparticles [27].

AF4 is one of the most promising techniques for the characterization of nanoparticles due to the high precision and wide separation range it provides, as well as the great variety of nanomaterials that can be analysed. Currently, this technique is in continuous progress and shows high versatility, because it has been used to characterize many types of nanomaterials such as nanodrugs, silica nanoparticles, nanocellulose and nanoplastics, among others [28-31]. AF4 is a chromatographic technique consisting of a laminar flow of a carrier liquid combined with a transverse flow. The induced cross-flow field interacts with any molecule or particle in the channel and, in this way, size separation occurs [32]. This phenomenon gives rise to different elution times, which are inversely proportional to the diffusion coefficients of the particles in the sample, that is, to the radius of gyration. This technique is able to separate particles with sizes ranging from $2 \mathrm{~nm}$ to $1 \mu \mathrm{m}$ [33]. Furthermore, it can be coupled with different types of detectors such as UV-Vis, multiangle light scattering (MALS) and/or dynamic light scattering (DLS) that would allow the complete characterization of different nanomaterials [34,35], and the determination of the molar mass distribution of macromolecules [36]. In addition, AF4 has been coupled online with ICP-MS for the selective detection of $\mathrm{TiO}_{2}$ nanoparticles [37] and in combination with UV-Vis spectroscopy and off-line HR-ICP-MS for the determination of Ag nanoparticles [38], rendering this technique very useful and versatile for analysing different types of materials.

Herein, we report the synthesis of unfunctionalized, non-oxidized and isolated graphene nanosheets (GNS) applying the ultrasound-assisted method by liquid-phase exfoliation of a micrographite. The nanosheets obtained have been characterized using different techniques, particularly related to the study of particle size. Thus, besides XRD, TEM and Raman spectroscopy, three techniques, specifically oriented to the determination of particle size, i.e., DLS, NTA and AF4, were investigated. The proper choice of the parent micrographite has been essential because it has provided GNS particles measurable by all these techniques. A comparative study of the results obtained with the different characterization techniques is reported.

\section{Materials and Methods}

\subsection{Materials}

Microcrystalline graphite powder (98\%) was purchased from Nanostructured \& Amorphous Materials Inc. (Katy, TX, USA). 1,2-dichlorobenzene (ODCB, anhydrous, 99\%), dichloromethane (anhydrous, $\geq 99.8 \%$, contains 40-150 ppm amylene as stabilizer) and lanthanum hexaboride (powder, $10 \mu \mathrm{m}, 99 \%$ ) were obtained from Sigma Aldrich (San Luis, MO, USA).

\subsection{Methods}

\subsubsection{Synthesis of Graphene Nanosheets (GNS)}

$250 \mathrm{mg}$ of microcrystalline graphite powder (MG) were stirred in $50 \mathrm{~mL}$ of $o$-dichlorobenzene (ODCB) for $30 \mathrm{~min}$. Subsequently, the resulting dispersion was subjected to treatment with a pulsed ultrasound probe for $2 \mathrm{~h}$ using an Ultrasonic Homogenizer 4710 Series from Cole Parmer Instrument Co. (Vernon Hills, IL, USA) (45\% amplitude, $60 \%$ duty cycle). The power supplied by the equipment was $9.96 \mathrm{~W}$, according to a calorimetric calibration method [39] (see SI and Figure S1). The suspension obtained was centrifuged at $1935 \times \mathrm{g}(4000 \mathrm{rpm})$ for $30 \mathrm{~min}$ to remove the non-exfoliated micrographite. The remaining suspension was again centrifuged at $12,096 \times g(10,000 \mathrm{rpm})$ to isolate the graphene nanosheets. They were washed three times with dichloromethane by successive redispersions and centrifugations to remove the ODCB. A Sorvall Super T-21 Tabletop Superspeed Centrifuge (Du Pont, Delaware, NJ, USA) was used to carry out all centrifugation processes. Dichloromethane was 
evaporated from the resulting graphene nanosheets dispersion at room temperature for $12 \mathrm{~h}$ and, finally, the powdered exfoliated GNS were dried under vacuum at $80^{\circ} \mathrm{C}$ overnight.

\subsubsection{X-ray Diffraction Analysis (XRD)}

X-ray diffraction patterns of the micrographite and graphene nanosheets were performed using a Bruker D8 Discover (Billerica, MA, USA) with a monochromatic $\mathrm{CuK} \alpha$ radiation source. The scanning conditions for structural analysis were an angular range of $15^{\circ}-80^{\circ}(2 \theta)$, a $0.040^{\circ}$ step size and $1.05 \mathrm{~s}$ per step. Additional measurements of X-ray diffraction were carried out using a Bruker D8 Advance. The scanning condition for this analysis were an angular range of $5^{\circ}-40^{\circ}(2 \theta)$, a $0.002^{\circ}$ step size and 9 s per step.

\subsubsection{Transmission Electron Microscopy}

Transmission electron microscopy (TEM) images were recorded on a Jeol JEM-1400 transmission electron microscope (Akishima, Tokyo, Japan) operated at an accelerating voltage of $120 \mathrm{kV}$. The instrument has a high-contrast objective-lens polepiece, enabling high-contrast TEM image observation at all magnifications and good image quality, as well as easy handling. In addition, it has an advanced vacuum system. The digital-camera configuration includes a CCD camera that enables focusing and image verification on the operation screen. The measurement CCD camera $(\mathrm{MC})$, orthogonal to the alignment camera, has a resolution of $1344 \times 1024$ pixel (12 bits/pixel). The measurements have been made in a magnification range between $8000 \times$ and $150,000 \times$ with an instrument resolution of $0.38 \mathrm{~nm}$ between points.

\subsubsection{Raman Spectroscopy}

Raman spectra were acquired with a Renishaw Raman instrument (InVia Raman Microscope, Wotton-under-Edge, UK) equipped with a Leica microscope furnished with various lenses, monochromators and filters in addition to a CCD camera. A silicon standard sample was used as reference for calibration $\left(520 \mathrm{~cm}^{-1}\right)$. Spectra were obtained by excitation with green laser light (532 nm) from 150 to $3500 \mathrm{~cm}^{-1}$. A total of 32 scans per spectrum were acquired to improve the signal-to-noise ratio.

\subsubsection{X-ray Photoelectron Spectroscopy (XPS)}

X-ray photoelectron spectroscopy (XPS) was performed through a SPECS mod. PHOIBOS 150 $\mathrm{MCD}$ spectrometer (Berlin, Germany) using non-monochromatic $\mathrm{Mg} \mathrm{K} \alpha$ radiation and a multichannel detector. All spectra were fitted to Gauss-Lorentz curves to better identify the different chemical environment of the elements in each material. The binding energy values were calibrated with the adventitious carbon C 1 s signal $(284.8 \mathrm{eV})$.

\subsubsection{Dynamic Light Scattering (DLS)}

Particle sizes were measured in a Zetasizer ZSP (Malvern Instrument Ltd., Worcestershire, UK) at $25{ }^{\circ} \mathrm{C}$ based on laser Doppler velocimetry and dynamic light scattering (DLS) techniques. Previously, the suspension was homogenized using an ultrasonication probe for a period of $5 \mathrm{~min}$.

The refraction index values were set at 1.33 and 2.38 for the dispersant (deionized water) and the material (carbon), respectively. The analysis was carried out by triplicate and medium and standard deviation were calculated. To obtain the hydrodynamic radius $\left(R_{h}\right)$ of the GNS particles, the hydrodynamic diameter $\left(D_{h}\right)$ was calculated by using the Stokes-Einstein Equation (1):

$$
D_{h}=\frac{K_{B} T}{3 \pi \eta_{0} D_{t}}
$$


where $K_{B}$ is the Boltzmann constant, $T$ the temperature in $K$ degrees, $\eta_{0}$ the solvent viscosity, and $D_{t}$ the translational diffusion coefficient.

The intensity size distribution or the Z-average diameter was obtained from the autocorrelation function using the "general purpose mode" for the materials.

\subsubsection{Nanoparticle Tracking Analysis (NTA)}

NTA measurements were performed in a NanoSight NS300 (Malvern Panalytical Ltd., Malvern, UK), equipped with a sample chamber and a $488 \mathrm{~nm}$ laser.

For the NTA measurements, $1 \mathrm{mg}$ of GNS was suspended in $1 \mathrm{~mL}$ of water. Before the analysis, the solution was homogenized for $3 \mathrm{~min}$ in an ultrasonic bath. The samples were injected into the chamber with sterile syringes. Many individual particles are tracked during their Brownian motion by the software, which uses the Stokes-Einstein Equation (2) to provide sample information:

$$
\overline{(x, y)}^{2}=\frac{2 K_{B} T}{3 R_{h} \pi \eta}
$$

where $K_{B}$ is the Boltzmann constant and $\overline{(x, y)}^{2}$ is the mean-squared speed of a particle with a hydrodynamic radius of $R_{h}$ in a medium of defined viscosity and temperature. All measurements were performed at room temperature.

\subsubsection{Asymmetric Flow Field Flow Fractionation (AF4)}

An AF4 system (AF2000, Postnova Analytics, Landsberg am Lech, Germany) coupled online to a 21-angle MALS detector (PN3621, Postnova Analytics, Landsberg am Lech, Germany) and UV-Vis diode array detector (PN3241 Postnova Analytics) was used. The MALS detectors were calibrated using bovine serum albumin monomer and detectors at different angles were normalized with respect to a $90^{\circ}$ detector measuring a sodium poly(styrenesulfonate) (PSS) standard. The AF4 channel was trapezoidal-shaped, $350 \mu \mathrm{m}$ thick (defined by a spacer), $29.8 \mathrm{~cm}$ long (inlet to outlet) and had a maximum width of $2 \mathrm{~cm}$. Particle recoveries over AF4 were tested with several commonly used membranes. The analytical parameters and characteristics of our AF4 system are presented in Table 1. All injections were carried out with an autosampler in triplicate (PN5300, Postnova Analytics).

Table 1. Analytical parameters and characteristics of asymmetric flow field flow fractionation (AF4).

\begin{tabular}{cc}
\hline AF4 Parameters & AF2000 System \\
\hline Membranes & CR, PAN, PVDF \\
Channel geometry & Trapezoidal \\
Spacer thickness & $350 \mu \mathrm{m}$ \\
Focusing time & $4 \mathrm{~min}$ \\
Elution time & $40 \mathrm{~min}$ \\
Detector flow & $0.5 \mathrm{~mL} / \mathrm{min}$ \\
Injection flow & $0.2 \mathrm{~mL} / \mathrm{min}$ \\
Cross flow & $1 \mathrm{~mL} / \mathrm{min}$ \\
UV-Vis & $254 \mathrm{~nm}$ \\
\hline
\end{tabular}

\section{Results}

\subsection{X-ray Diffraction (XRD)}

The structural properties of micrographite (MG) and graphene nanosheets (GNS) samples were examined by XRD. Figure 1 shows the XRD patterns, which revealed the characteristic peaks for these materials. Both samples exhibited a strong peak at ca. $26.5^{\circ}(2 \theta)$, which corresponded to the (002) graphite reflection [40]. Also, a weak peak at $54.5^{\circ}(2 \theta)$, attributed to the (004) reflection, was present in the samples, especially in the micrographite. The absence of the graphite peak for graphene oxide 
(GO) at $11^{\circ}(2 \theta)$ [41] confirmed that the graphene nanosheets preparation method did not cause partial oxidation of the starting graphite, as shown in the inserted image in Figure 1 . The higher relative intensity of the graphite signals indicated a higher crystallinity compared to the GNS. The full widths at half-maximum of the (002) peak (FWHM) were studied to determine the crystallite size (LaB $6 \mathrm{Was}$ used as a pattern to determine the instrumental broadening). FWHM for GNS was higher than for MG (see Table 2). The crystallite size, L, was calculated through the Scherrer's Equation (3):

$$
\mathrm{L}=\frac{\mathrm{k} \lambda}{\beta \cos \theta}
$$

where $\lambda$ is the $X$-ray wavelength in nanometer $(\mathrm{nm}), \beta$ is the peak width of the diffraction peak profile at half maximum height in radians, $\theta$ is the scattering angle in radians and $\mathrm{k}$ is a constant related to crystallite shape, taken as 0.9 for the (002) Bragg reflection.

The instrumental broadening effect on FWHM was subtracted out using Warren's method on the assumption of a Gaussian peak [42]:

$$
\beta^{2}=\beta_{\text {sample }}^{2}-\beta_{\text {instrumental }}^{2}
$$

where $\beta_{\text {instrumental }}$ is referred to $\mathrm{LaB}_{6}$ and equal to 0.130 .

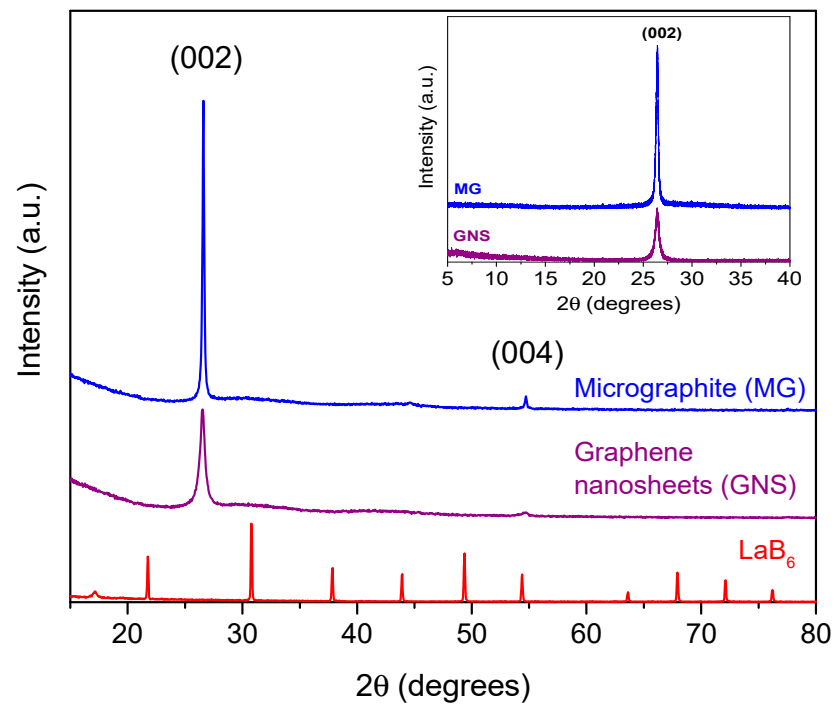

Figure 1. XRD patterns of micrographite (MG), graphene nanosheets (GNS) and LaB6 samples. Inset: XRD patterns of MG and GNS recorded from $5^{\circ}$ to $40^{\circ}(2 \theta)$.

Table 2. Structural parameters of the studied materials derived from their XRD patterns.

\begin{tabular}{ccccc}
\hline Samples & $\begin{array}{c}\text { Peak } \\
{ }^{\circ}(\mathbf{2 \theta})\end{array}$ & $\begin{array}{c}\mathbf{d}_{\mathbf{0 0 2}} \\
(\mathbf{A})\end{array}$ & FWHM & $\begin{array}{c}\text { Crystallite Size * } \\
\text { (nm) }\end{array}$ \\
\hline $\begin{array}{c}\text { Micrographite } \\
\begin{array}{c}\text { Graphene } \\
\text { nanosheets }\end{array}\end{array}$ & 26.6 & 3.346 & 0.171 & 73.3 \\
\hline \multicolumn{5}{c}{ * Parameter calculated considering the instrumental broadening. }
\end{tabular}

Liquid-phase exfoliation by ultrasound-assisted synthesis for obtaining graphene nanosheets (GNS) produced smaller crystallite sizes. Moreover, the thickness of the crystallites along c axis decreased because the full width at half-maximum (FWHM) of the peak (002) was higher than that of the starting micrographite (MG) [42,43]. Thus, the action of ultrasonic waves generates a cavitation energy that caused an increase in the interlayer spacing $\left(\mathrm{d}_{002}\right)$ due to the exfoliation of the MG [44]. 
In this way, the GNS sample had a smaller crystallite size and a lower average stacking height of the layered structure, confirming an effective exfoliation of the graphite by the ultrasound probe treatment.

\subsection{Transmission Electronic Microscopy (TEM)}

The surface morphology of the original graphite and the synthesized graphene was examined by TEM images. Nanometric particles of graphite flakes are shown in Figure 2A-C. Graphene nanosheets (GNS) were observed as transparent layers (see Figure 2D-F). When graphene sheets folded over themselves, cross-sectional and a few layers with nanometric size 200-500 nm could be viewed. The folded graphene sheets that were placed parallel to the electron beam were observed as several dark lines. However, TEM images for graphite showed a dark spot due to the ordered stacking of a large number of layers. Also, it was verified that the size of the graphite particles were several times larger than GNS particles [40].
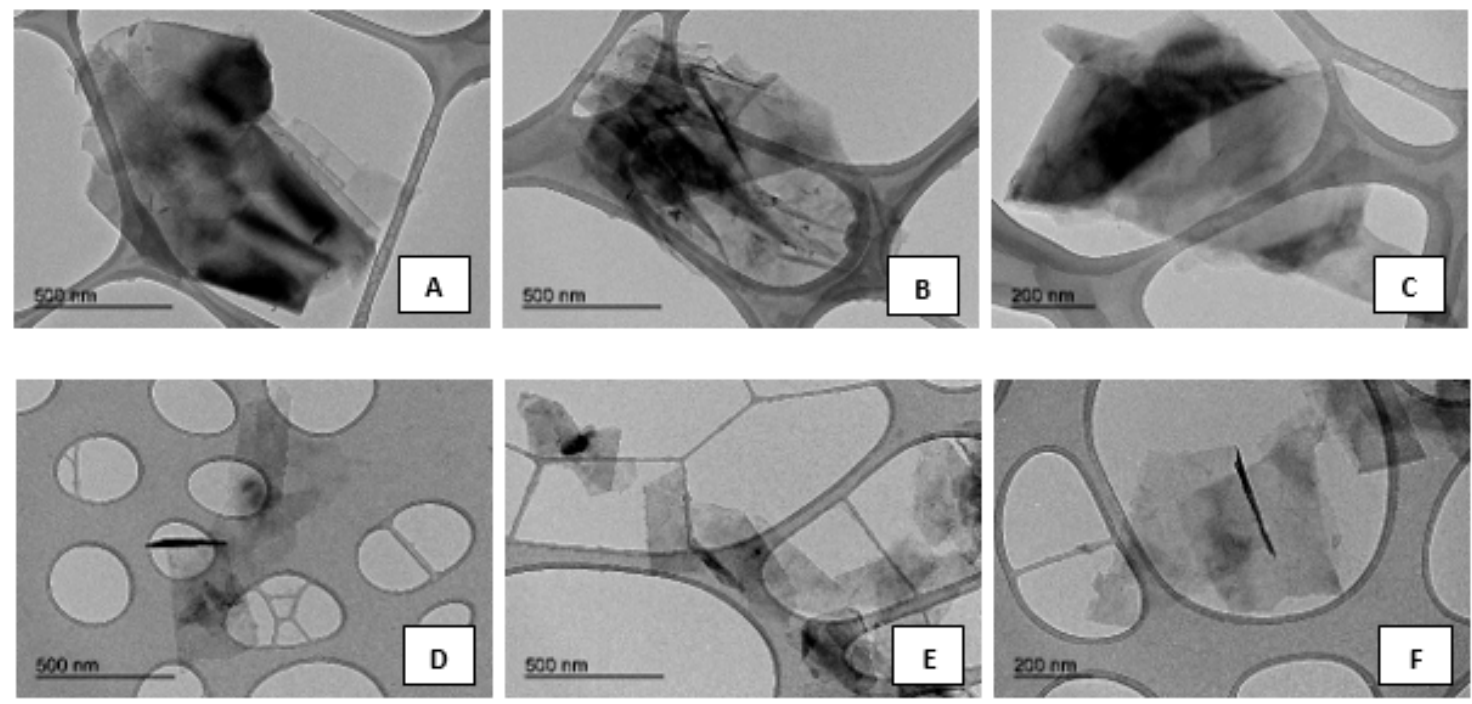

Figure 2. TEM images with different magnification for (A-C) micrographite (MG) and (D-F) graphene nanosheets (GNS) samples.

\subsection{Raman Spectroscopy}

Raman spectroscopy was performed to confirm the exfoliation of the micrographite by ultrasound irradiation. The typical bands of graphene materials were observed in the spectra (Figure 3). The G peak corresponds to the phonons of the energy level $E_{2 g}$ in the Brillouin zone. The intensity of the D band indicates the degree of disorder that the material possesses, which is related to several factors such as functionalization in the graphene macromolecular $\mathrm{sp}^{2}$ ring or, in our case, the ultrasonic exfoliation in ODCB. In many studies, the ratio of $\mathrm{I}_{\mathrm{D}} / \mathrm{I}_{\mathrm{G}}$ intensities is used to verify that the material has undergone a structural change during the synthesis process [45]. In addition, it has already been reported that this ratio is related to the flake size. Thus, an increase in the $\mathrm{I}_{\mathrm{D}} / \mathrm{I}_{\mathrm{G}}$ ratio indicates a smaller sheet size because the ratio of edge defects is greater for smaller layers [46]. The reference MG had $\mathrm{I}_{\mathrm{D}} / \mathrm{I}_{\mathrm{G}}=0.10$ while this ratio increased to 0.38 for the exfoliated GNS. These values confirmed the exfoliation of the graphite in ODCB using the ultrasound probe, which caused a certain disorder in the material. 


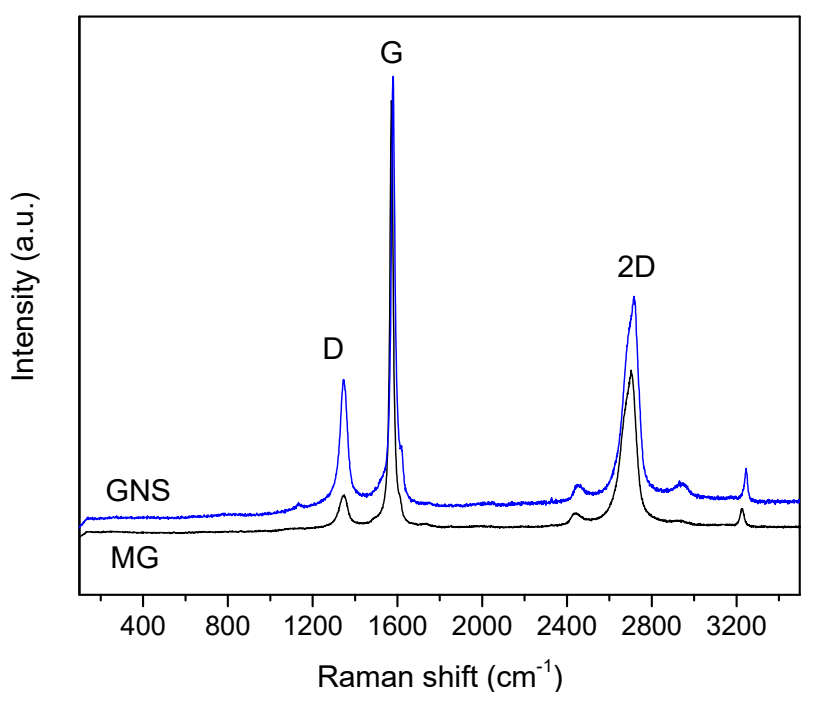

Figure 3. Raman spectra of MG and GNS samples.

The calculation of the number of graphene layers is very relevant in this type of study. Many investigations relate the position of the $G$ peak with the number of graphene layers. The $G$ peak of the GNS was at a Raman shift of $1582 \mathrm{~cm}^{-1}$ and so, applying the equation proposed by Wang et al. [47], it can be deduced that this material consisted of approximately seven layers of graphene nanosheets. The 2D peak was very remarkable, which is the overtone of the peak $\mathrm{D}$. The position, the shape of the line and the intensity of the peak are related to the number of layers [48]. Normally, a single layer of graphene shows a band at $2679 \mathrm{~cm}^{-1}$. It has been shown that in the case of multilayers graphene, the 2D peak shifts toward higher Raman shift and becomes broader [49]. In the case of GNS, the 2D band was centred at $2716 \mathrm{~cm}^{-1}$, similar to MG, although there was a clear difference in peak intensity between them. It is common to calculate the ratio of peak intensity $\mathrm{I}_{2 \mathrm{D}} / \mathrm{I}_{\mathrm{G}}$. An approximate value of 2 identifies a single layer of graphene [50]. The original micrographite showed an intensity ratio of 0.38 while the graphene nanosheets obtained after the ultrasonication process had a higher ratio of 0.55 , thus confirming the exfoliation of graphite again.

\subsection{X-ray Photoelectron Spectroscopy (XPS)}

In order to study the composition of MG and GNS, XPS spectra of these samples were measured. Figure 4a shows the XPS survey for the MG and GNS samples. Both materials were composed of $C$ and $\mathrm{O}$. The presence of $\mathrm{Al}$ is due to the holder where samples were placed. The oxygen content in the original graphite is in accordance with the purity of the sample and the presence of noncovalently bonded adsorbed oxygen [51]. The C/O ratio for GNS and MG samples, which is inserted in this figure, confirmed that the GNS material underwent a very slight oxidation upon exfoliation even though the difference between the MG and GNS ratios was almost negligible and insignificant compared to a common graphene oxide (GO) [52]. The X-ray photoelectron spectra in the $\mathrm{C} 1 \mathrm{~s}$ region of $\mathrm{MG}$ (Figure $4 \mathrm{~b}$ ) and GNS (Figure 4c) were fitted by overlapped peaks attributed to $\mathrm{C}-\mathrm{C}$ bonds $(284.8 \mathrm{eV})$, C-O bonds in hydroxy $(286.1 \mathrm{eV})$, epoxy $(287.7 \mathrm{eV})$, carbonyl $(289.1 \mathrm{eV})$ and carboxyl $(290.6 \mathrm{eV})$ groups, and the $\pi \rightarrow \pi^{*}$ transition (shake-up satellite peak at $292.3 \mathrm{eV}$ ) [53]. Therefore, no obvious changes were observed on the chemical structure of pristine MG and GNS obtained by the ultrasound-assisted method according to the $\mathrm{C} 1 \mathrm{~s}$ spectra. Therefore, it is confirmed that this method of synthesis allows obtaining GNS directly from MG, thus avoiding the synthesis of graphitic oxide (GO). The contribution of each component is summarized in Table 3. The values obtained for both samples are very similar, concluding that this method of exfoliation of the micrographite by the ultrasound treatment causes a delamination of the pristine graphite avoiding oxidation processes. These results are in agreement 
with those reported for graphene nanosheets prepared via ultrasonic exfoliation of graphite powder in $N$-methyl-2-pyrrolidone [54].
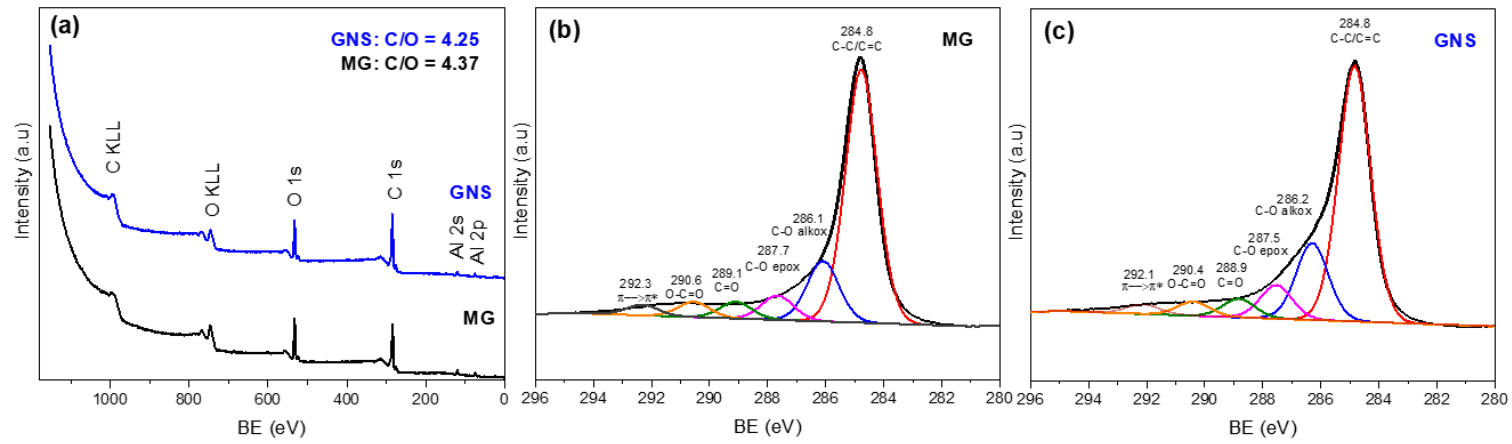

Figure 4. (a) XPS survey for MG and GNS and XPS spectra for the C 1s photoemission peak of (b) MG and (c) GNS.

Table 3. Contribution of the six components used in fitting of the $C$ 1s photoemission peak (in \%).

\begin{tabular}{ccccccc}
\hline Samples & C-C/C=C & C-O alkox & C-O epox & C=O & O-C=O & $\pi \rightarrow \pi^{*}$ \\
\hline (a) MG & 65.7 & 16.9 & 6.4 & 4.3 & 3.9 & 2.8 \\
(b) GNS & 64.2 & 18.2 & 8.1 & 4.1 & 3.5 & 1.9 \\
\hline
\end{tabular}

\subsection{Particle Size Distribution by DLS}

The size distribution and intensity correlation function normalized between one and zero of the GNS using dynamic light scattering (DLS) are shown in Figure 5. The advantage of applying this technique is its versatility in terms of fast, easy, reproducible and non-destructive results. DLS provides the hydrodynamic radius $\left(R_{h}\right)$, which is defined as the radius of an equivalent hard sphere diffusing at the same rate as the particle under observation, and so it is indicative of the apparent size adopted by the GNS in the aqueous dispersion. GNS exhibited a Gaussian peak located at $450 \pm 14 \mathrm{~nm}$. The measurement carried out for the MG material revealed a particle size outside the quantification limits of this technique $(>1 \mu \mathrm{m})$.
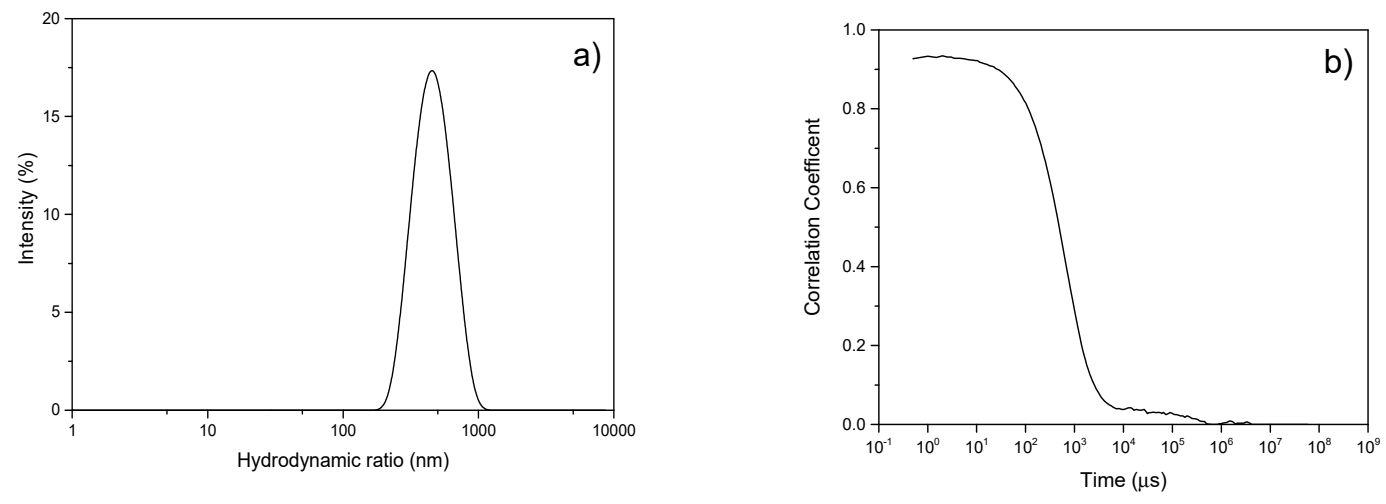

Figure 5. (a) Dynamic light scattering (DLS) size distribution curve of GNS and (b) normalized intensity correlation function of the scattered light intensity of GNS.

DLS also provides the polydispersity index (PDI), which indicates the width of the particle size distribution, being calculated as (peak width/peak height) ${ }^{2}$. A value of $\mathrm{PDI}<0.1$ indicates that the sample is monodisperse. Instead, if PDI is between $0.1<\mathrm{PDI}<0.2$, the sample would have a narrow particle size distribution. In the case of GNS, PDI was 0.36 , value between $0.2<\mathrm{PDI}<0.5$, which indicates that the sample has a wide particle size distribution according to the results reported by $\mathrm{J}$. Lohrke et al. [55]. 


\subsection{Particle Size Distribution by NTA}

In order to further define the size of the graphene nanosheets, nanoparticle tracking analysis (NTA) was used (Figure 6). This technique provides individual particle sizing, intensity distribution and approximate concentrations (Figure S2). Different particle sizes ranging from 40 to $400 \mathrm{~nm}$ were observed. The average size of three measurements gave a value of $151.8 \pm 12.7 \mathrm{~nm}$ and mean concentration of $1.02 \times 10^{9} \pm 6.48 \times 10^{7}$ particles $/ \mathrm{g}$. However, most particles had sizes between ca. 30-200 nm, although the concentration of particles around $300 \mathrm{~nm}$ was also significant. The number of larger particles (>350 nm) was much lower. A comparison between DLS and NTA reveals that DLS could overestimate the particle sizes of the sample. Both techniques provide a hydrodynamic radius $\left(R_{h}\right)$ value but larger particles scatter stronger and are preferably detected by DLS than the smaller particles since the scattering intensity effect depends on size rather than on the number of particles with similar diameter. DLS is a fast, simple and non-destructive technique, but it presents this inherent feature when analysing samples that present some polydispersity [56]. In these cases, it shows distributions shifted to the largest particle sizes. NTA, on the other hand, distinguishes with a greater precision the distribution of sizes $(10-2000 \mathrm{~nm})$ because it is based on the tracking of individual particles (Figure S3) [27].

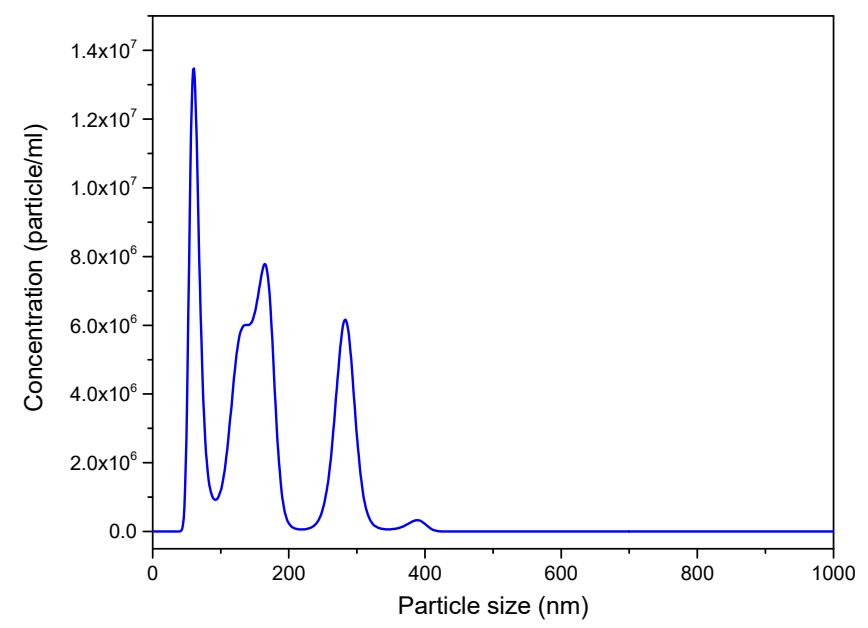

Figure 6. Nanoparticle tracking analysis (NTA) size distribution curve of GNS.

\subsection{Particle Size Distribution by AF4}

Firstly, the influence of the membrane composition on retention properties and recovery from the AF4 channel was studied. Polyvinylidene fluoride (PVDF), polyacrylonitrile (PAN) and regenerated cellulose (RC) were used. Figure 7 shows the AF4 fractograms using different membranes and the frequently used 0.2\% Novachem (Postnova Analytics) as carrier. Novachem is an aqueous surfactant mixture that stabilizes nanoparticles in solution.

The recovery from AF4 runs was determined as follows:

$$
\mathrm{R}(\%)=\frac{\mathrm{S}}{\mathrm{S}_{0}} \cdot 100
$$

where $S$ and $S_{0}$ are the peak areas without void peak of the detector signal (UV, $\lambda=254 \mathrm{~nm} / \mathrm{MALS}^{\circ}$ ), obtained with and without cross-flow, respectively. The MALS detector allows the direct measurement of the radius of gyration in the 1-1000 nm particle size range [57].

The fractograms $\left(90^{\circ}\right.$ light scattering detector) showed a low recovery using PAN and PVDF (less than $30 \%$ ) due to the retention of graphene nanosheets in these membranes. It was easily visualized because they became black. This had to be due to the interaction of the graphene sheets with these 
polymeric membranes. However, the fractogram exhibited a broad peak centred at around $33 \mathrm{~min}$ using a RC with a recovery of $30 \%$, thus suggesting a weaker interaction with this membrane.

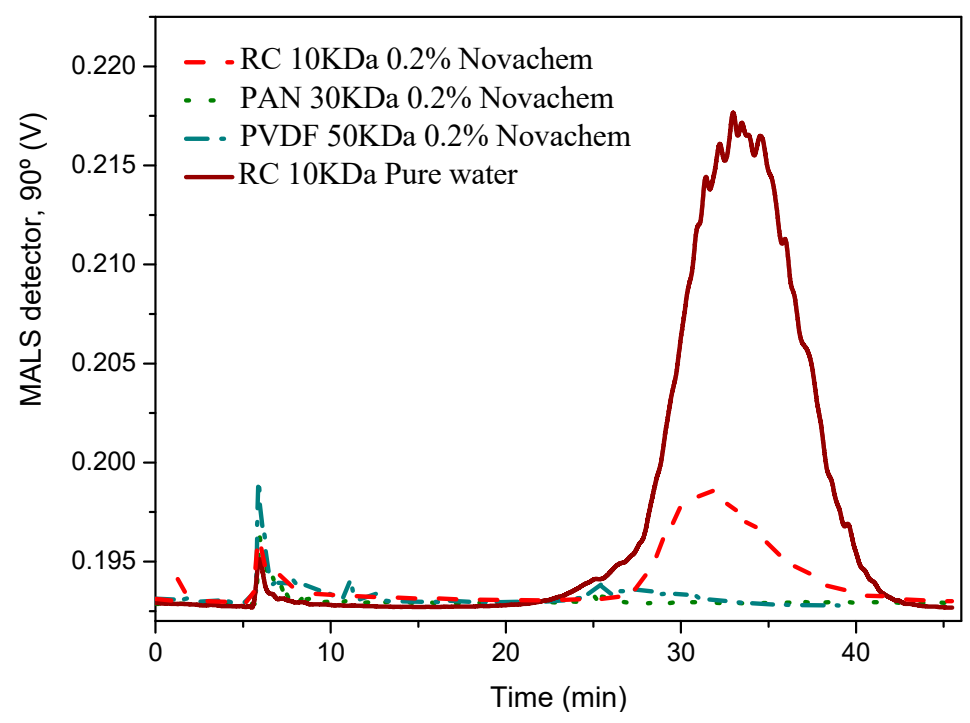

Figure 7. Asymmetric flow field flow fractionation (AF4) fractograms of GNS using regenerated cellulose (RC), polyacrylonitrile (PAN) and Polyvinylidene fluoride (PVDF) as membranes and Novachem and water as carriers.

To increase the recovery, pure water was essayed as carrier. Figure 7 shows an AF4 fractogram of GNS using a RC membrane, which resulted in a recovery of $95 \%$. These results indicated that there was no interaction between the membrane and the sample, and that water was the appropriate carrier to separate GNS by AF4.

The size distribution derived from the MALS detector showed the presence of different populations of particles (Figure 8). The most satisfactory fitting was achieved with the random coil model. This model is usually chosen for this particle range with all the different angles of the MALS detector providing robustness to the method (Figure S4). This detector provides the radius of gyration $\left(\mathrm{R}_{\mathrm{g}}\right)$, which is defined as the mass weighted average distance from the core of a particle to each mass element in the particle. $R_{g}$ is more dependent on the structure of the particle than the value of $R_{h}$. $R_{g}$ increases with increasing the elution volume. Figure 8 shows the size distribution (radius of gyration in $\mathrm{nm}$ ) as a function of the differential and cumulative distribution of the sample. The cumulative distribution indicates the fraction of a sample that has a mass in a certain range. Based on this, it is possible to determine GNS sample fractions simply by observing the height of the step. This parameter is provided by the differential distribution, which is calculated taking the differential of the cumulative distribution. In this way, it visually provides the weight fraction of the sample within a certain mass range, thus giving information on the resolution of the fractionation system and the analysis. The main peak of the fractogram was centred at around $360 \pm 11 \mathrm{~nm}$. As can be observed, this technique is able to discriminate populations of particles with similar sizes, thus allowing a better characterization of the graphene nanosheets.

MALS detector coupled to AF4 can provide relevant information about the mechanism of aggregation and nanoparticle structure. This information is included in the fractal dimension structural parameter $\left(D_{f}\right)$. $D_{f}$ gives a detailed information of the shape of the materials, thus allowing to understand the distribution of particles in aqueous media. A sphere has a $D_{f}$ of 3 , whereas $D_{f}$ is 2 for a planar structure and 1 for a rigid rod [58,59]. In the case of GNS, the calculated value was approximately 2 (1.85), which corresponded to 2-D objects with plain surface (Figure S5), thus confirming the regular layer structure observed by TEM. 


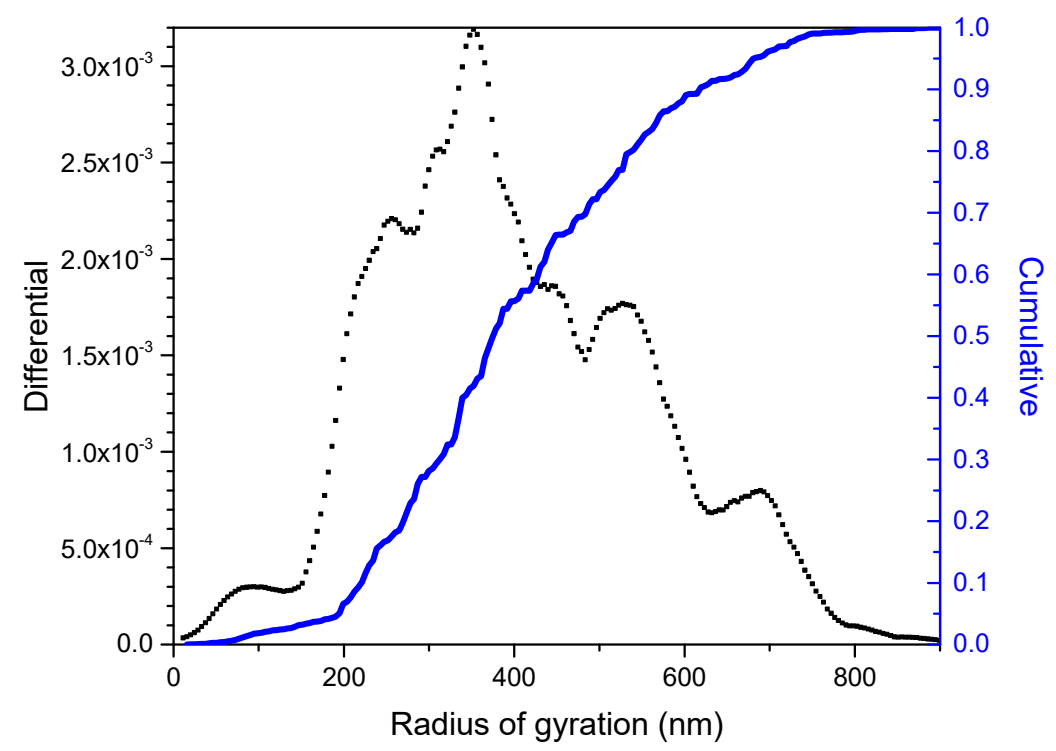

Figure 8. AF4 size distribution of GNS with a RC membrane using water as carrier.

Also, additional information on the particle shape can be obtained by calculating the shape factor, $p=R_{g} / R_{h}$, where $R_{g}$ is the average radius of gyration facilitated by AF4 $(360 \mathrm{~nm})$ and $R_{h}$ is the $Z$-average hydrodynamic radius provided by DLS $(450 \mathrm{~nm})$. The theoretical value of $\mathrm{p}$ for a hard sphere is 0.778 and for a stiff rod of approximately 2 [60]. The shape factor increases as the particle deviates from an ideal homogeneous spherical shape to a prolate $(\mathrm{p}>1)$ or oblate $(0.775<\mathrm{p}<1)$ ellipsoid [61,62]. The shape factor for GNS obtained by exfoliation of micrographite (MG) was 0.80 , thus indicating that the GNS particles have an oblate ellipsoid shape. The ability of AF4 to separate and fractionate GNS particles can be used to minimize the polydispersity of graphene preparations and so to obtain more accurate data on size and shape of these materials in future investigations.

In summary, AF4 and NTA give much richer information on particle size than DLS when dealing with a polydisperse material as graphene nanosheets. AF4 can be considered a promising technique for the separation and characterization of a variety of graphene structures, including not only graphene nanosheets, as described in this work, but also graphene oxide, graphene quantum dots and other carbon nanostructures. Unlike DLS and NTA, AF4 is a separation technique and can be coupled to a great variety of detectors, thus gathering rich information from the sample. It separates particles in the range from $1 \mathrm{~nm}$ to $1-2 \mu \mathrm{m}$, although other field flow fractionation techniques could expand this range up to particle sizes higher than $100 \mu \mathrm{m}$. Interestingly, the existence of semipreparative channels would allow the separation and collection of milligram quantities of these graphene structures. In addition, NTA can provide the concentration of GNS nanoparticles. Nevertheless, stability concerns of aqueous dispersions of large graphene particles, a more limited range of particle size determination and the current limited availability in many laboratories are among the disadvantages of NTA and AF4 relative to DLS.

\section{Conclusions}

The ultrasound-assisted exfoliation of micrographite (MG) to graphene nanosheets (GNS) was confirmed by different techniques, such as XRD, TEM (sheets size between 200-500 nm) and Raman spectroscopy. The particle size distribution of GNS was firstly determined by DLS (ca. $450 \mathrm{~nm}$ ), which provided the hydrodynamic radius and polydispersity of GNS. Subsequently, two different techniques, i.e., NTA and AF4, were applied here for the first time to graphene. NTA provided an average size of ca. $150 \mathrm{~nm}$ and higher resolution than DLS as well as approximate concentrations. In addition, it proved that DLS gave an average particle size whose intensity was biased towards the larger graphene particles. AF4 was able to separate various populations of GNS with different radius of gyration 
(centered at approx. $360 \mathrm{~nm}$ ) and in addition it provided information concerning the structure and shape of the particles. Clearly, the application of NTA and AF4 besides DLS allows gathering much rich information on graphene particles.

The above-mentioned advantages for NTA and AF4 reveal their usefulness for the determination of the actual polydispersity in graphene preparations, evaluation of their synthetic procedures and size determination and fractionation in aqueous media for studies related to biological applications, sensing and toxicity.

Supplementary Materials: The following are available online at http:/ /www.mdpi.com/2079-4991/9/2/152/s1, Figure S1: Calorimetric curve and linear fitting for the operational calibration of the ultrasonic equipment used for the GNS synthesis; Figure S2: NTA graph that represents GNS particle size and intensity; Figure S3: Nanoparticle tracking analysis images of GNS; Figure S4: Random Coil data fitting method; Figure S5: Analysis of the fractal factor dimension to identify the GNS particle shape where $M$ is molecular weight and $r$ is the radius of gyration.

Author Contributions: Conceptualization, A.C. and F.J.R.-S.; investigation, J.A.-G., A.B. and R.O.; writing-original draft preparation, J.A.-G. and A.B.; writing-review and editing, A.C., D.E. and F.J.R.-S.; supervision, C.J.-S. and J.M.

Funding: The authors wish to acknowledge the financial support from Ramon Areces Foundation, Spanish Ministry of Economy and Competitiveness (Project MAT2013-44463-R, MAT2017-87541-R), Spanish Ministry of Education, Culture and Sport for a FPU teaching and research fellowship (FPU17/03981), Andalusian Regional Government (FQM-346 and FQM-175 groups), Feder Funds, the support of Evelin Moldenhauer from Postnova Analytics $\mathrm{GmbH}$ and the technical staff from the Instituto Universitario de Investigación en Química Fina y Nanoquímica (IUIQFN).

Conflicts of Interest: The authors declare no conflict of interest.

\section{References}

1. Bhattacharjee, S. DLS and zeta potential-What they are and what they are not? J. Control. Release 2016, 235, 337-351. [CrossRef] [PubMed]

2. Gioria, S.; Caputo, F.; Urbán, P.; Maguire, C.M.; Bremer-Hoffmann, S.; Prina-Mello, A.; Calzolai, L.; Mehn, D. Are existing standard methods suitable for the evaluation of nanomedicines: Some case studies. Nanomedicine 2018, 13, 539-554. [CrossRef] [PubMed]

3. Omar, J.; Boix, A.; Kerckhove, G.; von Holst, C. Optimisation of asymmetric flow field-flow fractionation for the characterisation of nanoparticles in coated polydisperse $\mathrm{TiO}_{2}$ with applications in food and feed. Food Addit. Contam. Part A 2016, 33, 1775-1784. [CrossRef] [PubMed]

4. Reschiglian, P.; Zattoni, A.; Roda, B.; Michelini, E.; Roda, A. Field-flow fractionation and biotechnology. Trends Biotechnol. 2005, 23, 475-483. [CrossRef] [PubMed]

5. Contado, C.; Pagnoni, A. $\mathrm{TiO}_{2}$ in Commercial Sunscreen Lotion: Flow Field-Flow Fractionation and ICP-AES Together for Size Analysis. Anal. Chem. 2008, 80, 7594-7608. [CrossRef] [PubMed]

6. Guazzo, R.; Gardin, C.; Bellin, G.; Sbricoli, L.; Ferroni, L.; Ludovichetti, F.; Piattelli, A.; Antoniac, I.; Bressan, E.; Zavan, B. Graphene-Based Nanomaterials for Tissue Engineering in the Dental Field. Nanomaterials 2018, 8, 349. [CrossRef] [PubMed]

7. Garg, R.; Dutta, N.; Choudhury, N. Work Function Engineering of Graphene. Nanomaterials 2014, 4, 267-300. [CrossRef] [PubMed]

8. Lv, P.; Tan, X.-W.; Yu, K.-H.; Zheng, R.-L.; Zheng, J.-J.; Wei, W. Super-elastic graphene/carbon nanotube aerogel: A novel thermal interface material with highly thermal transport properties. Carbon N. Y. 2016, 99, 222-228. [CrossRef]

9. Suslick, K.S. Acoustic Cavitation in Homogeneous Liquids. Science 1990, 247, 1439-1447. [CrossRef]

10. Coleman, J.N. Liquid exfoliation of defect-free graphene. Acc. Chem. Res. 2013, 46, 14-22. [CrossRef]

11. Bourlinos, A.B.; Georgakilas, V.; Zboril, R.; Sterioti, T.A.; Stubos, A.K. Liquid-Phase Exfoliation of Graphite Towards Solubilized Graphenes. Small 2009, 5, 1841-1845. [CrossRef] [PubMed]

12. Hernandez, Y.; Nicolosi, V.; Lotya, M.; Blighe, F.M.; Sun, Z.; De, S.; McGovern, I.T.; Holland, B.; Byrne, M.; Gun'Ko, Y.K.; et al. High-yield production of graphene by liquid-phase exfoliation of graphite. Nat. Nanotechnol. 2008, 3, 563-568. [CrossRef] [PubMed]

13. Wei, P.; Shen, J.; Wu, K.; Hu, C. Tuning electrochemical behaviors of N-methyl-2-pyrrolidone liquid exfoliated graphene nanosheets by centrifugal speed-based grading. Carbon N. Y. 2018, 129, 183-190. [CrossRef] 
14. Hamilton, C.E.; Lomeda, J.R.; Sun, Z.; Tour, J.M.; Barron, A.R. High-yield organic dispersions of unfunctionalized graphene. Nano Lett. 2009, 9, 3460-3462. [CrossRef] [PubMed]

15. Łoś, S.; Duclaux, L.; Alvarez, L.; Hawełek, Ł.; Duber, S.; Kempiński, W. Cleavage and size reduction of graphite crystal using ultrasound radiation. Carbon N. Y. 2013, 55, 53-61. [CrossRef]

16. Quintana, M.; Grzelczak, M.; Spyrou, K.; Kooi, B.; Bals, S.; Van Tendeloo, G.; Rudolf, P.; Prato, M. Production of large graphene sheets by exfoliation of graphite under high power ultrasound in the presence of tiopronin. Chem. Commun. 2012, 48, 12159. [CrossRef] [PubMed]

17. Arao, Y.; Mori, F.; Kubouchi, M. Efficient solvent systems for improving production of few-layer graphene in liquid phase exfoliation. Carbon N. Y. 2017, 118, 18-24. [CrossRef]

18. Bahr, J.L.; Mickelson, E.T.; Bronikowski, M.J.; Smalley, R.E.; Tour, J.M. Dissolution of small diameter single-wall carbon nanotubes in organic solvents? Chem. Commun. 2001, 193-194. [CrossRef]

19. Qiu, L.-G.; Li, Z.-Q.; Wu, Y.; Wang, W.; Xu, T.; Jiang, X. Facile synthesis of nanocrystals of a microporous metal-organic framework by an ultrasonic method and selective sensing of organoamines. Chem. Commun. 2008, 3642. [CrossRef]

20. Báez, D.; Pardo, H.; Laborda, I.; Marco, J.; Yáñez, C.; Bollo, S. Reduced Graphene Oxides: Influence of the Reduction Method on the Electrocatalytic Effect towards Nucleic Acid Oxidation. Nanomaterials 2017, 7, 168. [CrossRef]

21. Lotya, M.; Rakovich, A.; Donegan, J.F.; Coleman, J.N. Measuring the lateral size of liquid-exfoliated nanosheets with dynamic light scattering. Nanotechnology 2013, 24, 265703. [CrossRef] [PubMed]

22. Badaire, S.; Poulin, P.; Maugey, M.; Zakri, C. In Situ Measurements of Nanotube Dimensions in Suspensions by Depolarized Dynamic Light Scattering. Langmuir 2004, 20, 10367-10370. [CrossRef] [PubMed]

23. Kato, H.; Nakamura, A.; Kinugasa, S. Effects of Angular Dependency of Particulate Light Scattering Intensity on Determination of Samples with Bimodal Size Distributions Using Dynamic Light Scattering Methods. Nanomaterials 2018, 8, 708. [CrossRef] [PubMed]

24. Walker, J.G. Improved nano-particle tracking analysis. Meas. Sci. Technol. 2012, 23, 065605. [CrossRef]

25. Dragovic, R.A.; Collett, G.P.; Hole, P.; Ferguson, D.J.P.; Redman, C.W.; Sargent, I.L.; Tannetta, D.S. Isolation of syncytiotrophoblast microvesicles and exosomes and their characterisation by multicolour flow cytometry and fluorescence Nanoparticle Tracking Analysis. Methods 2015, 87, 64-74. [CrossRef] [PubMed]

26. Ribeiro, L.N.D.M.; Couto, V.M.; Fraceto, L.F.; de Paula, E. Use of nanoparticle concentration as a tool to understand the structural properties of colloids. Sci. Rep. 2018, 8, 982. [CrossRef]

27. Filipe, V.; Hawe, A.; Jiskoot, W. Critical evaluation of nanoparticle tracking analysis (NTA) by NanoSight for the measurement of nanoparticles and protein aggregates. Pharm. Res. 2010, 27, 796-810. [CrossRef]

28. Espinosa, E.; Sánchez, R.; Otero, R.; Domínguez-Robles, J.; Rodríguez, A. A comparative study of the suitability of different cereal straws for lignocellulose nanofibers isolation. Int. J. Biol. Macromol. 2017, 103, 990-999. [CrossRef]

29. Ruiz-Palomero, C.; Laura Soriano, M.; Valcárcel, M. Detection of nanocellulose in commercial products and its size characterization using asymmetric flow field-flow fractionation. Microchim. Acta 2017, 184, 1069-1076. [CrossRef]

30. Bartczak, D.; Vincent, P.; Goenaga-Infante, H. Determination of Size- and Number-Based Concentration of Silica Nanoparticles in a Complex Biological Matrix by Online Techniques. Anal. Chem. 2015, 87, 5482-5485. [CrossRef]

31. Correia, M.; Loeschner, K. Detection of nanoplastics in food by asymmetric flow field-flow fractionation coupled to multi-angle light scattering: Possibilities, challenges and analytical limitations. Anal. Bioanal. Chem. 2018, 1-13. [CrossRef] [PubMed]

32. Korgel, B.A.; Van Zanten, J.H.; Monbouquette, H.G. Vesicle size distributions measured by flow field-flow fractionation coupled with multiangle light scattering. Biophys. J. 1998, 74, 3264-3272. [CrossRef]

33. Iavicoli, P.; Urbán, P.; Bella, A.; Ryadnov, M.G.; Rossi, F.; Calzolai, L. Application of Asymmetric Flow Field-Flow Fractionation hyphenations for liposome-antimicrobial peptide interaction. J. Chromatogr. A 2015, 1422, 260-269. [CrossRef] [PubMed]

34. Bednar, A.J.; Poda, A.R.; Mitrano, D.M.; Kennedy, A.J.; Gray, E.P.; Ranville, J.F.; Hayes, C.A.; Crocker, F.H.; Steevens, J.A. Comparison of on-line detectors for field flow fractionation analysis of nanomaterials. Talanta 2013, 104, 140-148. [CrossRef] [PubMed] 
35. Kato, H.; Nakamura, A.; Takahashi, K.; Kinugasa, S. Accurate Size and Size-Distribution Determination of Polystyrene Latex Nanoparticles in Aqueous Medium Using Dynamic Light Scattering and Asymmetrical Flow Field Flow Fractionation with Multi-Angle Light Scattering. Nanomaterials 2012, 2, 15-30. [CrossRef] [PubMed]

36. Choi, J.; Zielke, C.; Nilsson, L.; Lee, S. Characterization of the molar mass distribution of macromolecules in beer for different mashing processes using asymmetric flow field-flow fractionation (AF4) coupled with multiple detectors. Anal. Bioanal. Chem. 2017, 409, 4551-4558. [CrossRef] [PubMed]

37. López-Heras, I.; Madrid, Y.; Cámara, C. Prospects and difficulties in $\mathrm{TiO}_{2}$ nanoparticles analysis in cosmetic and food products using asymmetrical flow field-flow fractionation hyphenated to inductively coupled plasma mass spectrometry. Talanta 2014, 124, 71-78. [CrossRef]

38. Koopmans, G.F.; Hiemstra, T.; Regelink, I.C.; Molleman, B.; Comans, R.N.J. Asymmetric flow field-flow fractionation of manufactured silver nanoparticles spiked into soil solution. J. Chromatogr. A 2015, 1392, 100-109. [CrossRef]

39. Taurozzi, J.S.; Hackley, V.A.; Wiesner, M.R. Ultrasonic dispersion of nanoparticles for environmental, health and safety assessment-Issues and recommendations. Nanotoxicology 2011, 5, 711-729. [CrossRef]

40. Simón, M.; Benítez, A.; Caballero, A.; Morales, J.; Vargas, O. Untreated Natural Graphite as a Graphene Source for High-Performance Li-Ion Batteries. Batteries 2018, 4, 13. [CrossRef]

41. Benítez, A.; Di Lecce, D.; Elia, G.A.; Caballero, Á.; Morales, J.; Hassoun, J. A Lithium-Ion Battery using a 3 D-Array Nanostructured Graphene-Sulfur Cathode and a Silicon Oxide-Based Anode. ChemSusChem 2018, 11, 1512-1520. [CrossRef] [PubMed]

42. Arrebola, J.C.; Caballero, A.; Hernán, L.; Morales, J. Graphitized Carbons of Variable Morphology and Crystallinity: A Comparative Study of Their Performance in Lithium Cells. J. Electrochem. Soc. 2009, 156, A986. [CrossRef]

43. Ivanov, A.V.; Maksimova, N.V.; Kamaev, A.O.; Malakho, A.P.; Avdeev, V.V. Influence of intercalation and exfoliation conditions on macrostructure and microstructure of exfoliated graphite. Mater. Lett. 2018, 228, 403-406. [CrossRef]

44. Htwe, Y.Z.N.; Chow, W.S.; Suda, Y.; Thant, A.A.; Mariatti, M. Effect of electrolytes and sonication times on the formation of graphene using an electrochemical exfoliation process. Appl. Surf. Sci. 2019, 469, 951-961. [CrossRef]

45. Mohiuddin, T.M.G.; Lombardo, A.; Nair, R.R.; Bonetti, A.; Savini, G.; Jalil, R.; Bonini, N.; Basko, D.M.; Galiotis, C.; Marzari, N.; et al. Uniaxial strain in graphene by Raman spectroscopy: G peak splitting, Grüneisen parameters, and sample orientation. Phys. Rev. B 2009, 79, 205433. [CrossRef]

46. Arao, Y.; Kubouchi, M. High-rate production of few-layer graphene by high-power probe sonication. Carbon N. Y. 2015, 95, 802-808. [CrossRef]

47. Wang, H.; Wang, Y.; Cao, X.; Feng, M.; Lan, G. Vibrational properties of graphene and graphene layers. J. Raman Spectrosc. 2009, 40, 1791-1796. [CrossRef]

48. Ferrari, A.C.; Meyer, J.C.; Scardaci, V.; Casiraghi, C.; Lazzeri, M.; Mauri, F.; Piscanec, S.; Jiang, D.; Novoselov, K.S.; Roth, S.; et al. Raman spectrum of graphene and graphene layers. Phys. Rev. Lett. 2006, 97, 1-4. [CrossRef]

49. Hu, M.; Yao, Z.; Wang, X. Characterization techniques for graphene-based materials in catalysis. AIMS Mater. Sci. 2017, 4, 755-788. [CrossRef]

50. Parobek, D.; Shenoy, G.; Zhou, F.; Peng, Z.; Ward, M.; Liu, H. Synthesizing and Characterizing Graphene via Raman Spectroscopy: An Upper-Level Undergraduate Experiment That Exposes Students to Raman Spectroscopy and a 2D Nanomaterial. J. Chem. Educ. 2016, 93, 1798-1803. [CrossRef]

51. Voiry, D.; Yang, J.; Kupferberg, J.; Fullon, R.; Lee, C.; Jeong, H.Y.; Shin, H.S.; Chhowalla, M. High-quality graphene via microwave reduction of solution-exfoliated graphene oxide. Science. 2016, 353, 1413-1416. [CrossRef] [PubMed]

52. Hernández-Rentero, C.; Vargas, O.; Caballero, A.; Morales, J.; Martín, F. Solvothermal-induced 3D graphene networks: Role played by the structural and textural properties on lithium storage. Electrochim. Acta 2016, 222, 914-920. [CrossRef]

53. Ganguly, A.; Sharma, S.; Papakonstantinou, P.; Hamilton, J. Probing the Thermal Deoxygenation of Graphene Oxide Using High-Resolution In Situ X-ray-Based Spectroscopies. J. Phys. Chem. C 2011, 115, 17009-17019. [CrossRef] 
54. Wei, P.; Gan, T.; Wu, K. N-methyl-2-pyrrolidone exfoliated graphene as highly sensitive analytical platform for carbendazim. Sens. Actuators B Chem. 2018, 274, 551-559. [CrossRef]

55. Lohrke, J.; Briel, A.; Mäder, K. Characterization of superparamagnetic iron oxide nanoparticles by asymmetrical flow-field-flow-fractionation. Nanomedicine 2008, 3, 437-452. [CrossRef] [PubMed]

56. Guidetti, G.; Cantelli, A.; Mazzaro, R.; Ortolani, L.; Morandi, V.; Montalti, M. Tracking graphene by fluorescence imaging: A tool for detecting multiple populations of graphene in solution. Nanoscale 2016, 8 , 8505-8511. [CrossRef] [PubMed]

57. Wyatt, P.J. Submicrometer particle sizing by multiangle light scattering following fractionation. J. Colloid Interface Sci. 1998, 197, 9-20. [CrossRef]

58. Gigault, J.; Grassl, B. Improving the understanding of fullerene (nC60) aggregate structures: Fractal dimension characterization by static light scattering coupled to asymmetrical flow field flow fractionation. J. Colloid Interface Sci. 2017, 502, 193-200. [CrossRef]

59. Raghavendra, B.S.; Dutt, D.N. Computing Fractal Dimension of Signals using Multiresolution Box-counting Method. Int. J. Electr. Comput. Energy Electron. Commun. Eng. 2010, 4, 50-65.

60. Mathaes, R.; Winter, G.; Engert, J.; Besheer, A. Application of different analytical methods for the characterization of non-spherical micro- And nanoparticles. Int. J. Pharm. 2013, 453, 620-629. [CrossRef]

61. Baalousha, M.; Kammer, F.V.D.; Motelica-Heino, M.; Hilal, H.S.; Le Coustumer, P. Size fractionation and characterization of natural colloids by flow-field flow fractionation coupled to multi-angle laser light scattering. J. Chromatogr. A 2006, 1104, 272-281. [CrossRef] [PubMed]

62. Gigault, J.; Le Hécho, I.; Dubascoux, S.; Potin-Gautier, M.; Lespes, G. Single walled carbon nanotube length determination by asymmetrical-flow field-flow fractionation hyphenated to multi-angle laser-light scattering. J. Chromatogr. A 2010, 1217, 7891-7897. [CrossRef] [PubMed]

(C) 2019 by the authors. Licensee MDPI, Basel, Switzerland. This article is an open access article distributed under the terms and conditions of the Creative Commons Attribution (CC BY) license (http:/ / creativecommons.org/licenses/by/4.0/). 